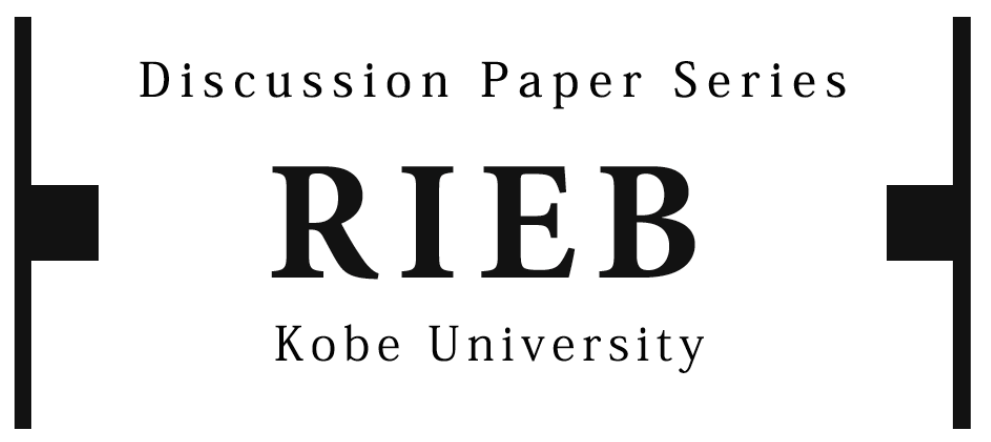

DP2018-10

Periodic Solutions of the One-sector Growth Model: The Role of Income Effects

Kazumichi IWASA

Gerhard SORGER

March 29, 2018

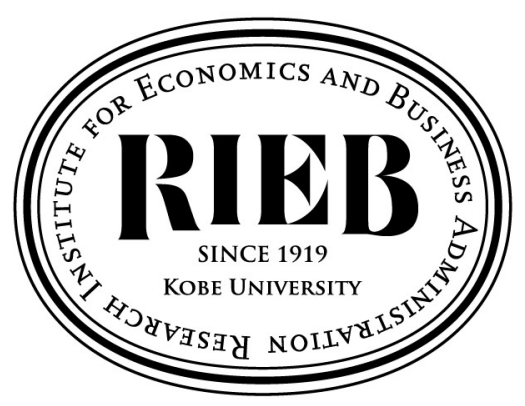

Research Institute for Economics and Business Administration Kobe University 


\title{
Periodic solutions of the one-sector growth model: the role of income effects
}

\author{
Kazumichi IWASA \\ Research Institute for Economics and Business Administration \\ Kobe University, Japan \\ Gerhard SORGER \\ Department of Economics \\ University of Vienna, Austria
}

March 29, 2018

\begin{abstract}
The discrete-time version of the neoclassical one-sector growth model with elastic labor supply is considered. It is shown that this model can have periodic solutions only if leisure is an inferior good.
\end{abstract}

Journal of Economic Literature classification codes: C61, O41

Key words: Optimal growth; elastic labor supply; periodic solutions; inferiority of leisure 


\section{Introduction}

The usual paradigm of business cycle research is that fluctuations of macroeconomic aggregates are caused by exogenous stochastic shocks to the economy. This is reflected by the most popular modelling approach for business cycles, the dynamic stochastic general equilibrium (DSGE) framework going back to [14] and [15]. On the other hand, there have been attempts to explain business cycles as a phenomenon that is endogenously generated by the market mechanism itself. Whereas the early literature on endogenous business cycles focussed on periodic solutions (see, e.g., [12], [11], [10], or [8]), later contributions such as [5] or [9] emphasized mechanisms leading to irregular or chaotic fluctuations (see [2] for a survey). Based on a careful examination of the spectral properties of macroeconomic time series, it has recently been argued that the periodic solutions proposed by the early literature may still be an important ingredient of actual business cycles; see [1]. In this regard it is worthwhile to mention that the non-stochastic version of even the most basic DSGE model allows for periodic solutions (see [6] and [19]) and it therefore becomes an important task to identify the mechanisms that generate these cycles. Whereas it is argued in [6] that substitution effects are primarily responsible for the occurrence of cycles, the results in [19] point to income effects instead. The purpose of the present note is to rigorously prove that income effects are necessary for the existence of periodic optimal solutions of the neoclassical one-sector model with elastic labor supply. More specifically, it is shown that whenever such a periodic optimal solution exists, leisure must be an inferior good at least on some parts of the domain of the utility function.

The proof of the main result in the present paper combines a characterization theorem from [19] with a graphical method that has been developed by [3]. It has to be emphasized that

the characterization theorem from [19] alone is not sufficient to prove that leisure needs to be inferior for periodic solutions to exist. This observation underlines the power of the graphical approach and shows that it forms an independent and methodological contribution. The rest of the paper is organized as follows. Section 2 formulates the model, states the assumptions, and presents the main result. The proof is contained in section 3. 


\section{Model formulation and result}

Time evolves in discrete periods $t \in \mathbb{N}_{0}=\{0,1,2, \ldots\}$. Let us denote by $k_{t}$ and $n_{t}$ the period- $t$ factor inputs of capital and labor, respectively, and by $f\left(k_{t}, n_{t}\right)$ the amount of output that is available in period $t$. This amount consists of output produced in period $t$ plus non-depreciated capital from the previous period. The production function $f$ satisfies the following assumption. ${ }^{1}$

Assumption 1 (i) The function $f: \mathbb{R}_{+} \times[0,1] \mapsto \mathbb{R}_{+}$is continuous, concave, homogeneous of degree 1 , and continuously differentiable on $\mathbb{R}_{++} \times(0,1]$.

(ii) There exists $\bar{k}>0$ such that $f(\bar{k}, 1)=\bar{k}$.

(iii) For every $k>0$ it holds that $f(k, n)$ is strictly increasing and strictly concave with respect to $n \in[0,1]$. Moreover, $\lim _{n \searrow 0} f_{2}(k, n)=+\infty$.

(iv) For every $n \in(0,1]$ it holds that $f(k, n)$ is strictly increasing and strictly concave with respect to $k \in \mathbb{R}_{+}$. Moreover, $\lim _{k \searrow 0} f_{1}(k, n)=+\infty$.

Output can be used for consumption and for investment. Denoting the amount that is consumed in period $t$ by $c_{t}$, this implies that

$$
c_{t}+k_{t+1}=f\left(k_{t}, n_{t}\right)
$$

holds for all $t \in \mathbb{N}_{0}$. The economy is endowed with a single unit of time per period such that

$$
\ell_{t}=1-n_{t}
$$

denotes the time that is available for leisure. The preferences of the social planner are described by the welfare functional

$$
\sum_{t=0}^{+\infty} \beta^{t} u\left(c_{t}, \ell_{t}\right),
$$

\footnotetext{
${ }^{1}$ Throughout the paper we denote partial derivatives by subscripts. For example, $f_{1}(k, n)$ is the partial derivative of the production function $f$ with respect to its first argument evaluated at the point $(k, n)$. Analogously, $f_{2}(k, n)$ is the partial derivative of the production function $f$ with respect to its second argument evaluated at the point $(k, n)$.
} 
where $u$ is an instantaneous utility function depending on consumption and leisure and where $\beta$ is a time-preference factor. ${ }^{2}$

Assumption 2 (i) The function $u: \mathbb{R}_{+} \times[0,1] \mapsto \mathbb{R}$ is continuous, concave, and twice continuously differentiable on $\mathbb{R}_{++} \times(0,1]$.

(ii) For every $c>0$ it holds that $u(c, \ell)$ is strictly increasing and strictly concave with respect to $\ell \in[0,1]$.

(iii) For every $\ell \in(0,1]$ it holds that $u(c, \ell)$ is strictly increasing and strictly concave with respect to $c \in \mathbb{R}$.

(iv) For all $(c, \ell) \in \mathbb{R}_{++} \times(0,1]$ it holds that $u_{11}(c, \ell) u_{22}(c, \ell)-u_{12}(c, \ell)^{2}>0$.

Assumption 3 It holds that $\beta \in(0,1)$.

An economy is a triple $(f, u, \beta)$. Suppose that an economy $(f, u, \beta)$ and an initial capital endowment $\kappa \in \mathbb{R}_{+}$are given. A sequence $\left(k_{t}, c_{t}, n_{t}, \ell_{t}\right)_{t=0}^{+\infty}$ is called a feasible allocation if conditions (1)-(2) and the non-negativity constraints $k_{t} \geq 0, c_{t} \geq 0, n_{t} \geq 0$, and $\ell_{t} \geq 0$ hold for all $t \in \mathbb{N}_{0}$. A feasible allocation $\left(k_{t}, c_{t}, n_{t}, \ell_{t}\right)_{t=0}^{+\infty}$ is said to be interior if $\left(k_{t}, c_{t}, \ell_{t}\right) \in \mathbb{R}_{++}^{2} \times(0,1)$ holds for all $t \in \mathbb{N}_{0}$, and it is said to be optimal from $\kappa$ if it maximizes the welfare functional (3) over all feasible allocations with the given initial capital endowment $k_{0}=\kappa$. A feasible allocation $\left(k_{t}, c_{t}, n_{t}, \ell_{t}\right)_{t=0}^{+\infty}$ is called an optimal allocation for the economy $(f, u, \beta)$ if there exists an initial endowment $\kappa \in \mathbb{R}_{+}$such that $\left(k_{t}, c_{t}, n_{t}, \ell_{t}\right)_{t=0}^{+\infty}$ is an optimal allocation from $\kappa$. Let an arbitrary integer $p \geq 2$ be given. A feasible allocation $\left(k_{t}, c_{t}, n_{t}, \ell_{t}\right)_{t=0}^{+\infty}$ is said to be periodic of period $p$ if the following two conditions hold for all $(t, s) \in \mathbb{N}_{0}^{2}$ :

$$
\begin{aligned}
& {[t \equiv s \bmod p] \Rightarrow\left(k_{t}, c_{t}, n_{t}, \ell_{t}\right)=\left(k_{s}, c_{s}, n_{s}, \ell_{s}\right),} \\
& {[t \not \equiv s \bmod p] \Rightarrow k_{t} \neq k_{s} .}
\end{aligned}
$$

It is known from [6] and [19] that there exist economies $(f, u, \beta)$ which admit periodic optimal allocations.

\footnotetext{
${ }^{2}$ We formulate the model as a social planner problem. One could also formulate it as a dynamic general equilibrium model with identical households and identical firms. Since the first welfare theorem applies to this model, equilibria of the dynamic general equilibrium model would coincide with optimal solutions of the social planner problem.
} 
Consider the static utility maximization problem

$$
\begin{array}{cc}
\operatorname{maximize}_{c, \ell} & u(c, \ell) \\
\text { subject to } & 0 \leq c, 0 \leq \ell, c+q \ell \leq I,
\end{array}
$$

where $I$ denotes income and $q$ the price of leisure in terms of consumption. Under assumption 2 this problem has a unique solution $(c(I, q), \ell(I, q))$ for all $q>0$ and all $I>0$. The income expansion path corresponding to the fixed price $q$ is the locus $\{(c(I, q), \ell(I, q)) \mid I>0\}$. Leisure is a normal good if the mapping $I \mapsto \ell(I, q)$ is non-decreasing for all $q>0$, and it is an inferior good otherwise. Analogous definitions apply to consumption.

We are now ready to formulate the main result of the present paper.

Theorem 1 Let $(f, u, \beta)$ be an economy satisfying assumptions 1 -3 and let $p \geq 2$ be a given integer. If the economy $(f, u, \beta)$ admits an optimal interior allocation which is periodic of period $p$, then it follows that leisure is an inferior good.

\section{Proof of theorem 1}

We start with a few preliminary considerations. Under assumptions 1-3, an interior feasible allocation is an optimal allocation if and only if the first-order optimality conditions

$$
\begin{aligned}
& u_{1}\left(c_{t}, \ell_{t}\right)=\beta f_{1}\left(k_{t+1}, n_{t+1}\right) u_{1}\left(c_{t+1}, \ell_{t+1}\right) \text { for all } t \in \mathbb{N}_{0}, \\
& u_{1}\left(c_{t}, \ell_{t}\right) f_{2}\left(k_{t}, n_{t}\right)=u_{2}\left(c_{t}, \ell_{t}\right) \text { for all } t \in \mathbb{N}_{0}
\end{aligned}
$$

as well as the transversality condition $\lim _{t \rightarrow+\infty} \beta^{t} u_{1}\left(c_{t}, \ell_{t}\right) k_{t+1}=0$ are satisfied; see [13] or [19]. Standard results from dynamic programming show that, for every initial capital endowment $\kappa \in[0, \bar{k}]$, there exists a unique optimal allocation from $\kappa$; see, e.g., [16], [18], or [20]. Moreover, there exists a continuous function $h:[0, \bar{k}] \mapsto[0, \bar{k}]$ such that the set of all capital sequences corresponding to optimal allocations for the economy $(f, u, \beta)$ coincides with the set of all trajectories of the difference equation

$$
k_{t+1}=h\left(k_{t}\right) \text { for all } t \in \mathbb{N}_{0}
$$


which start in initial states $k_{0} \in[0, \bar{k}]$. This result says that the capital stocks in every optimal allocation form a trajectory of a continuous dynamical system defined on the one-dimensional and compact state space $[0, \bar{k}]$. These systems are very well-studied and it is known that the existence of a periodic solution of any period $p \geq 3$ implies that there exists a periodic solution with period 2 as well; see, e.g., [4], [7], [17], or [18]. As a consequence, every economy $(f, u, \beta)$ that admits an optimal allocation of period $p \geq 3$ must also admit an optimal allocation of period 2. It is therefore sufficient to prove the inferiority of leisure under the assumption that there exists a periodic optimal allocation of period $p=2$. The following lemma summarizes necessary and sufficient optimality conditions for this case.

Lemma 1 (a) Let $(f, u, \beta)$ be an economy satisfying assumptions 1-3 and suppose that there exist real numbers $k_{a}, k_{b}, c_{a}, c_{b}, n_{a}, n_{b}, \ell_{a}$, and $\ell_{b}$ such that $k_{a} \neq k_{b}$ and $\left(k_{i}, c_{i}, n_{i}, \ell_{i}\right) \in$ $\mathbb{R}_{++}^{2} \times(0,1)^{2}$ for $i \in\{a, b\}$ hold. The sequence $\left(k_{t}, c_{t}, n_{t}, \ell_{t}\right)_{t=0}^{+\infty}$ defined by

$$
\left(k_{t}, c_{t}, n_{t}, \ell_{t}\right)= \begin{cases}\left(k_{a}, c_{a}, n_{a}, \ell_{a}\right) & \text { if } t \equiv 0 \bmod 2, \\ \left(k_{b}, c_{b}, n_{b}, \ell_{b}\right) & \text { if } t \equiv 1 \bmod 2\end{cases}
$$

is an optimal allocation for the economy $(f, u, \beta)$ if and only if the following conditions hold:

$$
\begin{aligned}
& n_{a}+\ell_{a}=n_{b}+\ell_{b}=1, \\
& c_{a}+k_{b}=f\left(k_{a}, n_{a}\right), \\
& c_{b}+k_{a}=f\left(k_{b}, n_{b}\right), \\
& u_{1}\left(c_{a}, \ell_{a}\right)=\beta f_{1}\left(k_{b}, n_{b}\right) u_{1}\left(c_{b}, \ell_{b}\right), \\
& u_{1}\left(c_{b}, \ell_{b}\right)=\beta f_{1}\left(k_{a}, n_{a}\right) u_{1}\left(c_{a}, \ell_{a}\right), \\
& u_{1}\left(c_{a}, \ell_{a}\right) f_{2}\left(k_{a}, n_{a}\right)=u_{2}\left(c_{a}, \ell_{a}\right), \\
& u_{1}\left(c_{b}, \ell_{b}\right) f_{2}\left(k_{b}, n_{b}\right)=u_{2}\left(c_{b}, \ell_{b}\right) .
\end{aligned}
$$

(b) Conditions (8)-(14) imply that $n_{a} \neq n_{b}$ and

$$
c_{b}-c_{a}+f_{2}\left(k_{a}, n_{a}\right)\left(n_{a}-n_{b}\right)>\beta f_{1}\left(k_{a}, n_{a}\right)\left[c_{b}-c_{a}+f_{2}\left(k_{b}, n_{b}\right)\left(n_{a}-n_{b}\right)\right] .
$$

Proof: (a) Consider the allocation specified by (7). Because of $\left(k_{i}, c_{i}, n_{i}, \ell_{i}\right) \in \mathbb{R}_{++}^{2} \times(0,1)^{2}$ the allocation is feasible and interior if and only if (8)-(10) hold. The Euler equation (4) holds 
along the given allocation if and only if (11)-(12) are satisfied, and the first-order condition (5) holds along the given allocation if and only if (13)-(14) are satisfied. Finally, the transversality condition holds because of the boundedness and interiority of the allocation and because of assumption 3. This completes the proof of part (a).

(b) From [19, equation (7)] it follows that

$$
\begin{aligned}
& f\left(k_{b}, n_{b}\right)+k_{b}-k_{a}-f_{1}\left(k_{a}, n_{a}\right) k_{a}-f_{2}\left(k_{a}, n_{a}\right) n_{b} \\
+ & \beta f_{1}\left(k_{a}, n_{a}\right)\left[f\left(k_{a}, n_{a}\right)+k_{a}-k_{b}-f_{1}\left(k_{b}, n_{b}\right) k_{b}-f_{2}\left(k_{b}, n_{b}\right) n_{a}\right]>0 .
\end{aligned}
$$

Using (9)-(10) and Euler's theorem for linearly homogeneous functions, this inequality can be expressed as (15).

Now suppose that $n_{a}=n_{b}=n$ holds. From (11)-(12) we obtain

$$
\beta^{2} f_{1}\left(k_{a}, n\right) f_{1}\left(k_{b}, n\right)=1
$$

and from (15) it follows that

$$
c_{b}-c_{a}>\beta f_{1}\left(k_{a}, n\right)\left(c_{b}-c_{a}\right) .
$$

These two conditions can only hold if either $c_{a}>c_{b}$ and $k_{a}<k_{b}$ or if $c_{a}<c_{b}$ and $k_{a}>k_{b}$ are satisfied. It is easy to see that neither of these possibilities is consistent with (9)-(10) and $n_{a}=n_{b}=n$. Hence, it follows that $n_{a} \neq n_{b}$ must be true.

The proof of theorem 1 is based on a graphical method developed in [3]. To introduce the reader to this graphical method let us use it to depict constant optimal allocations (steady state solutions). Suppose that $\left(k_{t}, c_{t}, n_{t}, \ell_{t}\right)_{t=0}^{+\infty}$ is a constant interior optimal allocation with $\left(k_{t}, c_{t}, n_{t}, \ell_{t}\right)=(k, c, n, \ell)$ for all $t \in \mathbb{N}_{0}$. We denote the capital-labor ratio along this allocation by $\gamma$, that is, $\gamma=k / n$. It follows from assumption 1 and equation (4) that $\gamma$ is uniquely determined by $\beta f_{1}(\gamma, 1)=1$. The feasibility conditions (1) and (2) together with the optimality condition (5) imply that

$$
\begin{aligned}
& f_{2}(\gamma, 1)=\frac{u_{2}(c, 1-n)}{u_{1}(c, 1-n)}, \\
& c=n[f(\gamma, 1)-\gamma] .
\end{aligned}
$$


Note that Euler's theorem for linearly homogeneous functions together with $f_{1}(\gamma, 1)=1 / \beta$ and $\beta \in(0,1)$ implies that

$$
f(\gamma, 1)-\gamma=f_{1}(\gamma, 1) \gamma+f_{2}(\gamma, 1)-\gamma=\frac{(1-\beta) \gamma}{\beta}+f_{2}(\gamma, 1)>f_{2}(\gamma, 1)>0
$$

Figure 1 shows the leisure-consumption space, that is, the domain of the utility function $u .^{3}$ The downward sloping line depicts the resource constraint (17), whereas the upward sloping curve depicts the income expansion path corresponding to the relative price of leisure and consumption defined by equation (16).

As income increases, the optimal consumption bundle moves along the income expansion path. This path is upward sloping when both consumption and leisure are normal goods. Hence, under normality, there must exist a unique intersection with the resource constraint (17). If leisure is inferior, multiple optimal steady states may exist or there may be no interior optimal steady states. These possibilities are illustrated in figure 2: the income expansion path i yields three optimal steady states, the income expansion path ii (drawn in green) generates a continuum of optimal steady states, and the income expansion path iii (drawn in red) results in no interior optimal steady state. The possibility of these scenarios has been proved in [13].

One can also use this graphical device to show that the optimal steady state must be unique if consumption is inferior; see path iv in figure 2. As a matter of fact, because $u$ is strictly quasi-concave, an income expansion path can intersect a given budget constraint only once. Obviously, this implies that the slope of an income expansion path corresponding to the relative price $f_{2}(\gamma, 1)$ (such as path iv) must be larger than the slope of the budget constraint, which is $-f_{2}(\gamma, 1)$; see again figure 2 .

Now let us turn to allocations with period 2 as considered in lemma 1. Without loss of generality we may assume that $n_{a} \geq n_{b}$. Because of lemma 1(b) this implies $n_{a}>n_{b}$. There are three cases to be considered.

\footnotetext{
${ }^{3}$ Although consumption $c$ is the first argument of the utility function and leisure $\ell$ is its second, we draw the domain of $u$ with $\ell$ on the horizontal axis and $c$ on the vertical one. The reason is that our main result demonstrates that leisure is necessarily inferior, which means that the income expansion path must be backward bending. Graphical representations of backward bending curves in the context of labor supply are typically drawn with labor on the horizontal axis and not on the vertical one. It is therefore our hope that following this convention makes our drawings easier to understand.
} 
CASE 1: $f_{1}\left(k_{a}, n_{a}\right)=f_{1}\left(k_{b}, n_{b}\right)$. Defining $\gamma_{a}=k_{a} / n_{a}$ and $\gamma_{b}=k_{b} / n_{b}$ it follows that $\gamma_{a}=\gamma_{b}=\gamma$ and $f_{2}\left(k_{a}, n_{a}\right)=f_{2}\left(k_{b}, n_{b}\right)=f_{2}(\gamma, 1)$. Moreover, from (11)-(12) we get

$$
u_{1}\left(c_{a}, \ell_{a}\right)=u_{1}\left(c_{b}, \ell_{b}\right)
$$

As shown in figure 3, the intersection of the resource constraint (17) and the line defined by $c=n f(\gamma, 1)-n_{a} \gamma$ occurs at $\ell_{a}=1-n_{a}$, while the intersection of (17) and the line defined by $c=n f(\gamma, 1)-n_{b} \gamma$ occurs at $\ell_{b}=1-n_{b}$. From $\gamma_{a}=\gamma_{b}=\gamma$ and conditions (8)-(10) it follows that the points $A$ and $B$ in the figure have the coordinates $\left(c_{a}, \ell_{a}\right)$ and $\left(c_{b}, \ell_{b}\right)$, respectively. For conditions (13) and (14) to be satisfied, the income expansion path corresponding to the relative price $f_{2}(\gamma, 1)$ has to pass through both of these points. Under assumption 2, however, the marginal utility of income must be strictly decreasing in income along any income expansion path. This would require that $u_{1}\left(c_{a}, \ell_{a}\right) \neq u_{1}\left(c_{b}, \ell_{b}\right)$, which is a contradiction to (18). Hence, this case cannot occur.

CASE 2: $f_{1}\left(k_{a}, n_{a}\right)>f_{1}\left(k_{b}, n_{b}\right)$. In this case it holds that $\gamma_{a}<\gamma<\gamma_{b}$ and, consequently,

$$
\begin{aligned}
& f_{1}\left(\gamma_{a}, 1\right)>f_{1}(\gamma, 1)>f_{1}\left(\gamma_{b}, 1\right), \\
& f_{2}\left(\gamma_{a}, 1\right)<f_{2}(\gamma, 1)<f_{2}\left(\gamma_{b}, 1\right) .
\end{aligned}
$$

Equations (9)-(10) can be rewritten as $c_{a}=n_{a} f\left(\gamma_{a}, 1\right)-n_{b} \gamma_{b}$ and $c_{b}=n_{b} f\left(\gamma_{b}, 1\right)-n_{a} \gamma_{a}$. Together with the above inequalities this shows that point $A$ moves down whereas point $B$ moves up (relative to the situation depicted in figure 3), as shown in figure 4. Because of (13)(14), the income expansion paths corresponding to the relative prices $f_{2}\left(\gamma_{a}, 1\right)$ and $f_{2}\left(\gamma_{b}, 1\right)$ must pass through points $A$ and $B$, respectively. Obviously, the income expansion path corresponding to the higher price $f_{2}\left(\gamma_{b}, 1\right)$ must be positioned to the left of or above the income expansion path corresponding to the lower price $f_{2}\left(\gamma_{a}, 1\right)$. There are two different ways how this can happen; see figures 5 and 6 . In the case illustrated in figure 5 leisure is inferior, whereas in figure 6 consumption is inferior. We are now going to show that the case depicted in figure 6 cannot occur.

Because of $\beta f_{1}\left(\gamma_{a}, 1\right)>1$ and $f_{2}\left(\gamma_{a}, 1\right)<f_{2}\left(\gamma_{b}, 1\right)$ it follows from (15) that

$$
\frac{c_{a}-c_{b}}{n_{a}-n_{b}}>f_{2}\left(\gamma_{b}, 1\right)>f_{2}\left(\gamma_{a}, 1\right)
$$


In terms of figure 6 , this says that the absolute value of the slope of the line connecting $A$ and $B$ is larger than both $f_{2}\left(\gamma_{a}, 1\right)$ and $f_{2}\left(\gamma_{b}, 1\right)$. Since the slopes of the two income expansion paths must be bounded below by $-f_{2}\left(\gamma_{a}, 1\right)$ and $-f_{2}\left(\gamma_{b}, 1\right)$, respectively, this is a contradiction.

CASE 3: $f_{1}\left(k_{a}, n_{a}\right)<f_{1}\left(k_{b}, n_{b}\right)$. In this case it holds that $\gamma_{a}>\gamma>\gamma_{b}$ and, consequently,

$$
\begin{aligned}
& f_{1}\left(\gamma_{a}, 1\right)<f_{1}(\gamma, 1)<f_{1}\left(\gamma_{b}, 1\right), \\
& f_{2}\left(\gamma_{a}, 1\right)>f_{2}(\gamma, 1)>f_{2}\left(\gamma_{b}, 1\right) .
\end{aligned}
$$

Together with condition (11) this implies that

$$
u_{1}\left(c_{a}, \ell_{a}\right)>u_{1}\left(c_{b}, \ell_{b}\right)
$$

Furthermore, it follows from the same arguments as in case 2 that point $A$ moves up and that point $B$ moves down (relative to the situation shown in figure 3); see figure 7 .

Let us denote the line segment connecting points $A$ and $B$ in figures 7 and 8 by $X$. We claim that leisure cannot be normal at all points $(c, \ell) \in X$. Suppose to the contrary that leisure is normal everywhere on $X$. In this case it must hold for all $(c, \ell) \in X$ that

$$
f_{2}\left(\gamma_{a}, 1\right) \geq \frac{u_{2}(c, \ell)}{u_{1}(c, \ell)}
$$

This is the case, because any income expansion path with a higher relative price than $f_{2}\left(\gamma_{a}, 1\right)$ cannot cross the line $X$. Furthermore, when leisure is normal, then indifference curves have to become steeper when $c$ is increased while $\ell$ is kept constant. Formally, this means that $\partial /(\partial c)\left[u_{2}(c, \ell) / u_{1}(c, \ell)\right]>0$, which is equivalent to

$$
\frac{u_{2}(c, \ell)}{u_{1}(c, \ell)}>\frac{u_{12}(c, \ell)}{u_{11}(c, \ell)}
$$

Putting the last two inequalities together it follows for all $(c, \ell) \in X$ that

$$
f_{2}\left(\gamma_{a}, 1\right)>\frac{u_{12}(c, \ell)}{u_{11}(c, \ell)}
$$

holds.

Now consider the blue curve in figure 8 , which is the locus defined by $u_{1}(c, \ell)=u_{1}\left(c_{b}, \ell_{b}\right)$. We shall refer to this curve as $R$. Obviously, it passes through point $B$. Moreover, because of (19) 
it follows that $R$ cannot intersect the line $\ell=\ell_{a}$ below point $A$, but must do so above point $A$. This, in turn, implies that there exists an intersection point of $R$ and $X$, at which the absolute value of the slope of $R$ is larger than $\left(c_{a}-c_{b}\right) /\left(n_{a}-n_{b}\right)$, which is the absolute value of the slope of $X$. In figure 8 this intersection point is labelled as $D$. From the definition of $R$ it follows that the slope of $R$ at any point $(c, \ell)$ is given by $-u_{12}(c, \ell) / u_{11}(c, \ell)$. Hence, for $(c, \ell)=D \in X$ it holds that

$$
\frac{u_{12}(c, \ell)}{u_{11}(c, \ell)} \geq \frac{c_{a}-c_{b}}{n_{a}-n_{b}}
$$

Finally note that $X$ is steeper than the green line in figure 8 , which is defined by $c=n f\left(\gamma_{a}, 1\right)-$ $n_{b} \gamma_{b}$. Combining this observation with the above inequality and utilizing Euler's theorem for linearly homogeneous functions again, we obtain

$$
\frac{u_{12}(c, \ell)}{u_{11}(c, \ell)} \geq \frac{c_{a}-c_{b}}{n_{a}-n_{b}}>f\left(\gamma_{a}, 1\right)>f_{2}\left(\gamma_{a}, 1\right)
$$

Since this is a contradiction to (20), our assumption that leisure is normal everywhere on $X$ cannot be true.

\section{References}

[1] P. Beaudry, D. Galizia, and F. Portier, "Putting the cycle back into business cycle analysis", NBER Working Paper 22825, 2016.

[2] M. Boldrin and M. Woodford, "Equilibrium models displaying endogenous fluctuations and chaos", Journal of Monetary Economics 25 (1990), 189-222.

[3] E. W. Bond, K. Iwasa, and K. Nishimura, "The dynamic Heckscher-Ohlin model: a diagrammatic analysis", International Journal of Economic Theory 8 (2012), 197-211.

[4] P. Collet and J.-P. Eckmann, Iterated Maps on the Interval as Dynamical Systems, Birkhäuser, 1980.

[5] R. H. Day, "Irregular growth cycles", American Economic Review 72 (1982), 406-414.

[6] P. De Hek, "An aggregative model of capital accumulation with leisure-dependent utility", Journal of Economic Dynamics and Control 23 (1998), 255-276. 
[7] W. De Melo and S. Van Strien, One-Dimensional Dynamics, Springer-Verlag, 1993.

[8] R. Goodwin, "The nonlinear accelerator and the persistence of business cycles", Econometrica 19 (1951), 1-17.

[9] J.-M. Grandmont, "On endogenous competitive business cycles", Econometricy 53 (1985), 995-1045.

[10] J. Hicks, A Contribution to the Theory of the Trade Cycle, Clarendon Press, 1950.

[11] N. Kaldor, "A model of the trade cycle", Economic Journal 50 (1940), 78-92.

[12] M. Kalecki, "A theory of the business cycle", Review of Economic Studies 4 (1937), 77-97.

[13] T. Kamihigashi, "Multiple interior steady states in the Ramsey model with elastic labor supply", International Journal of Economic Theory 11 (2015), 25-37.

[14] F. E. Kydland and E. C. Prescott, "Time to build and aggregate fluctuations", Econometrica 50 (1982), 1345-1370.

[15] J. B. Long, Jr., and C. I. Plosser, "Real business cycles", Journal of Political Economy 91 (1983), 39-69.

[16] J. Miao, Economic Dynamics in Discrete Time, MIT Press, 2014.

[17] A. N. Sarkovskii, "Coexistence of cycles of a continuous map of a line into itself", Ukrains'kyi Matematychnyi Zhurnal 16 (1964), 61-71.

[18] G. Sorger, Dynamic Economic Analysis: Deterministic Models in Discrete Time, Cambridge University Press, 2015.

[19] G. Sorger, "Cycles and chaos in the one-sector growth model with elastic labor supply", Economic Theory (2017), forthcoming.

[20] N. Stokey and R. E. Lucas Jr, Recursive Methods in Economic Dynamics, Harvard University Press, 1989. 


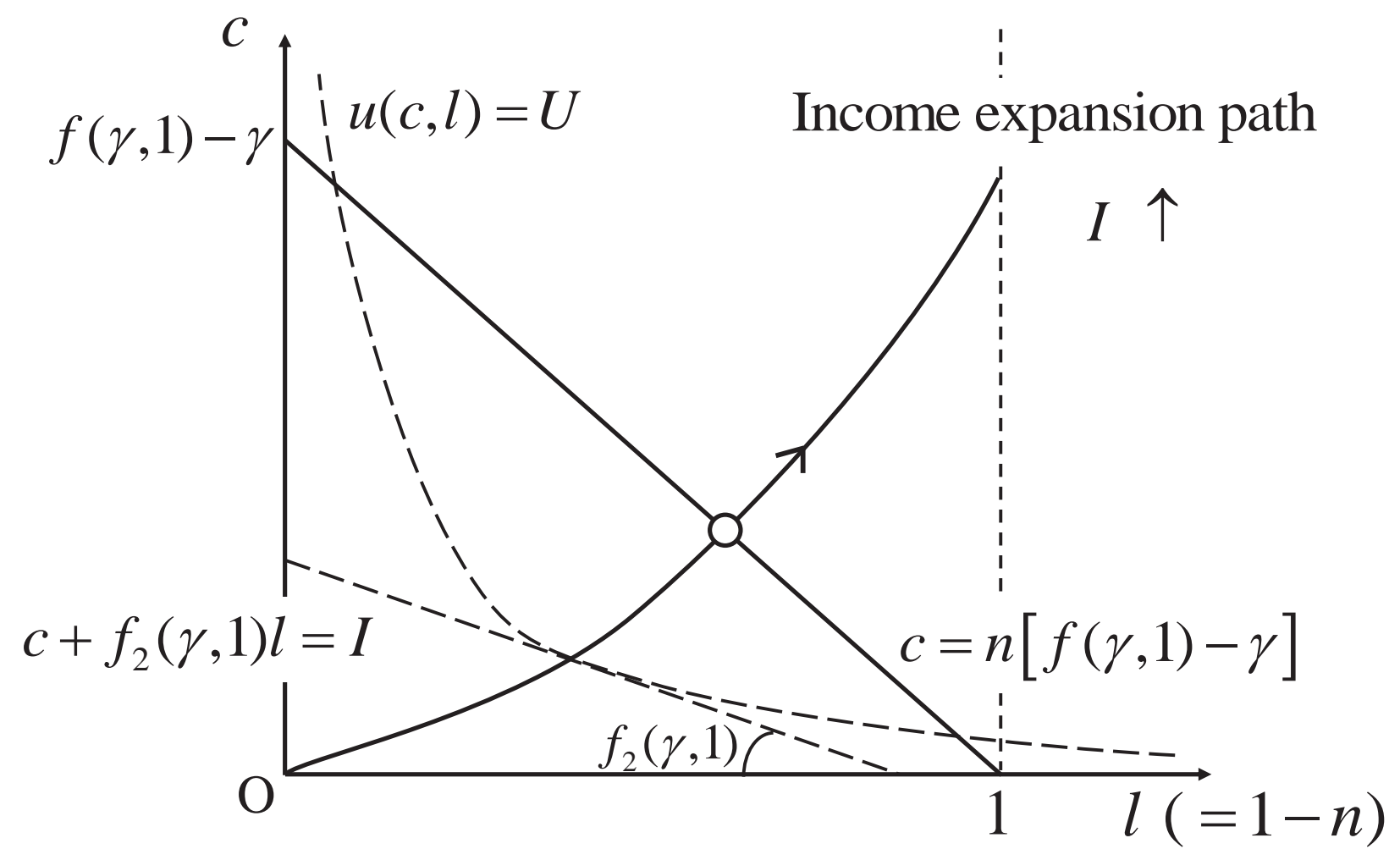

Figure 1: The income expansion path and the steady state

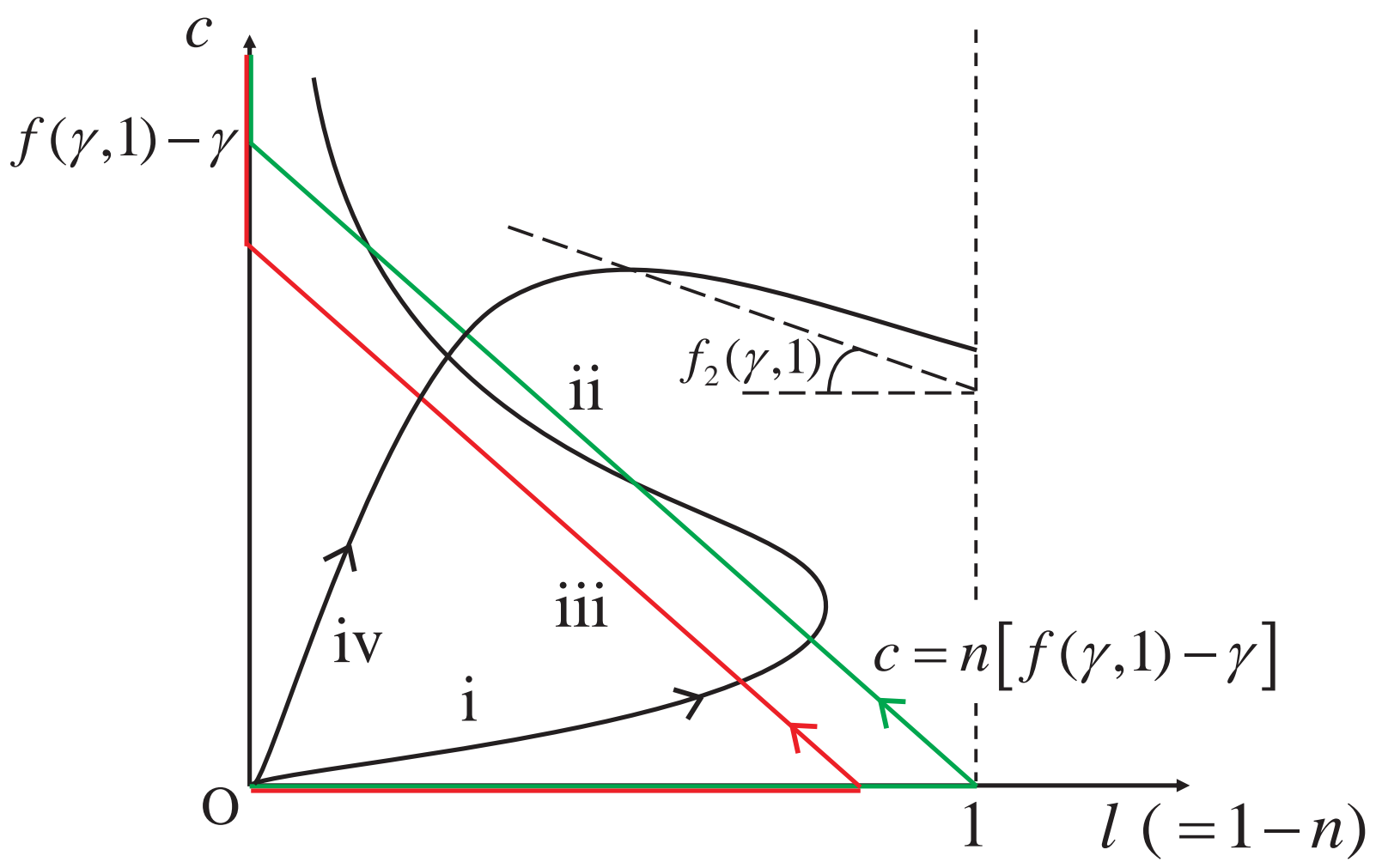

Figure 2: Inferiority and multiple steady states 


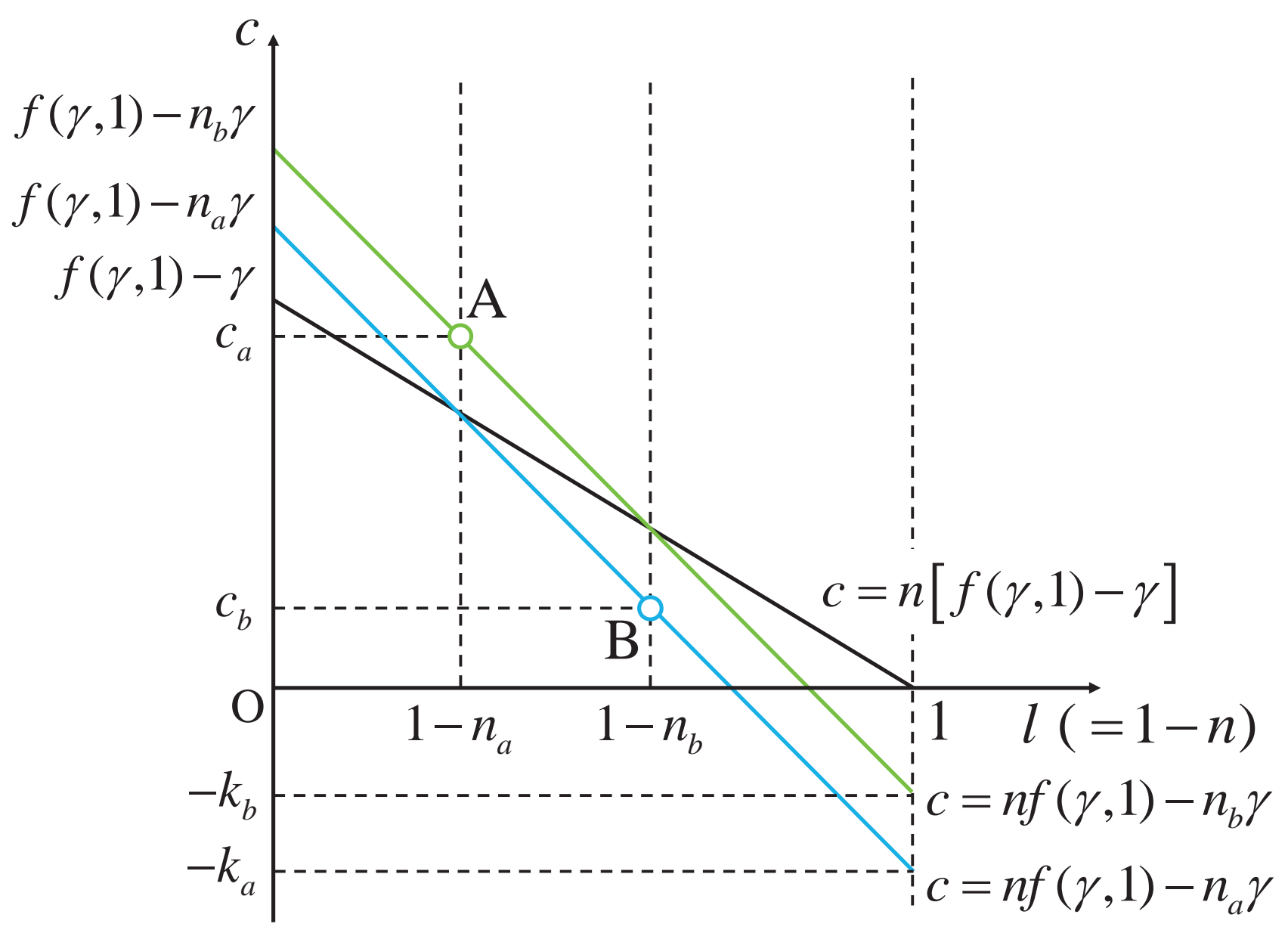

Figure 3: Pairs $\left(c_{a}, l_{a}\right)$ and $\left(c_{b}, l_{b}\right)$ with $\gamma_{a}=\gamma=\gamma_{b}$

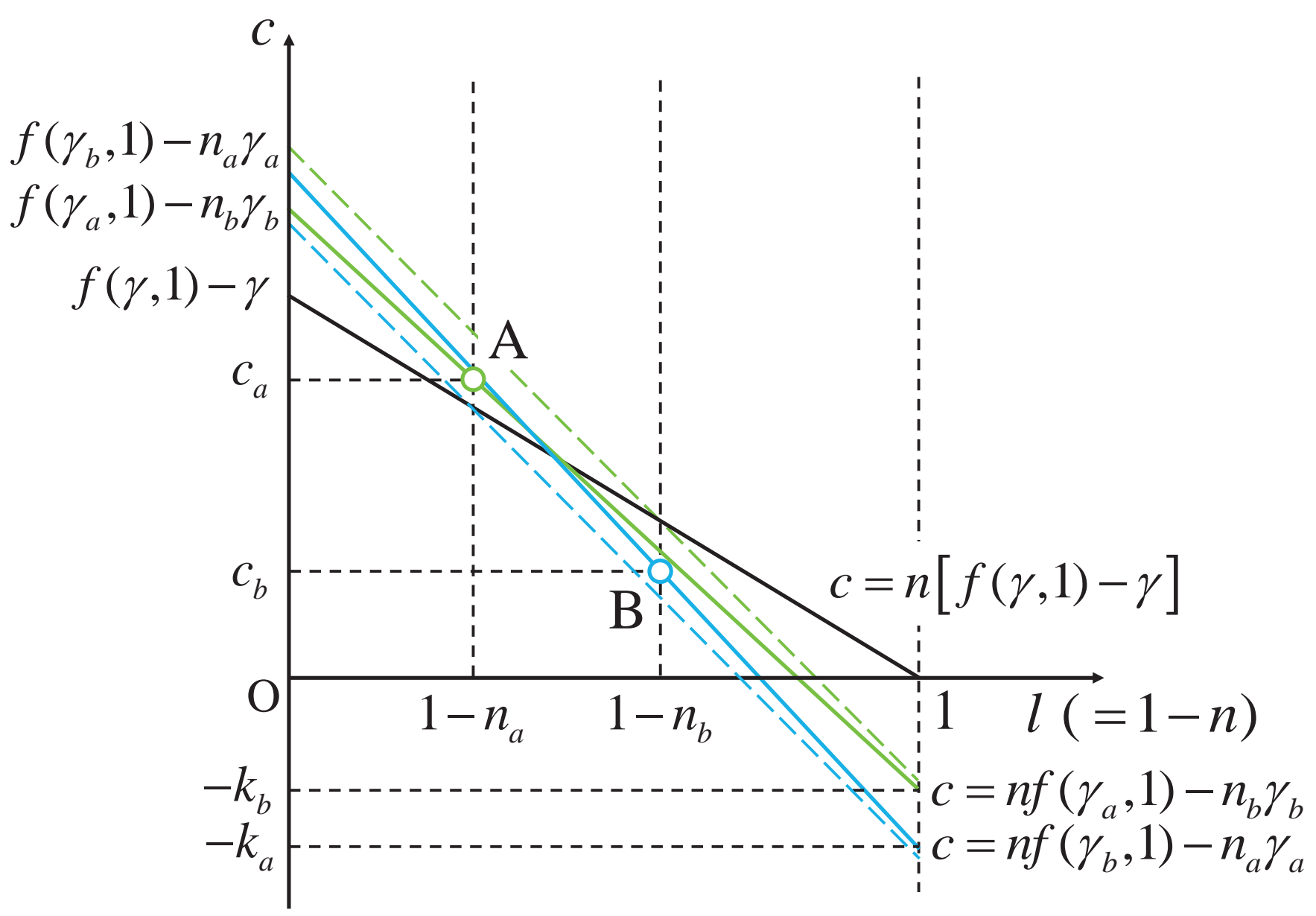

Figure 4: Pairs $\left(c_{a}, l_{a}\right)$ and $\left(c_{b}, l_{b}\right)$ with $\gamma_{a}<\gamma<\gamma_{b}$ 


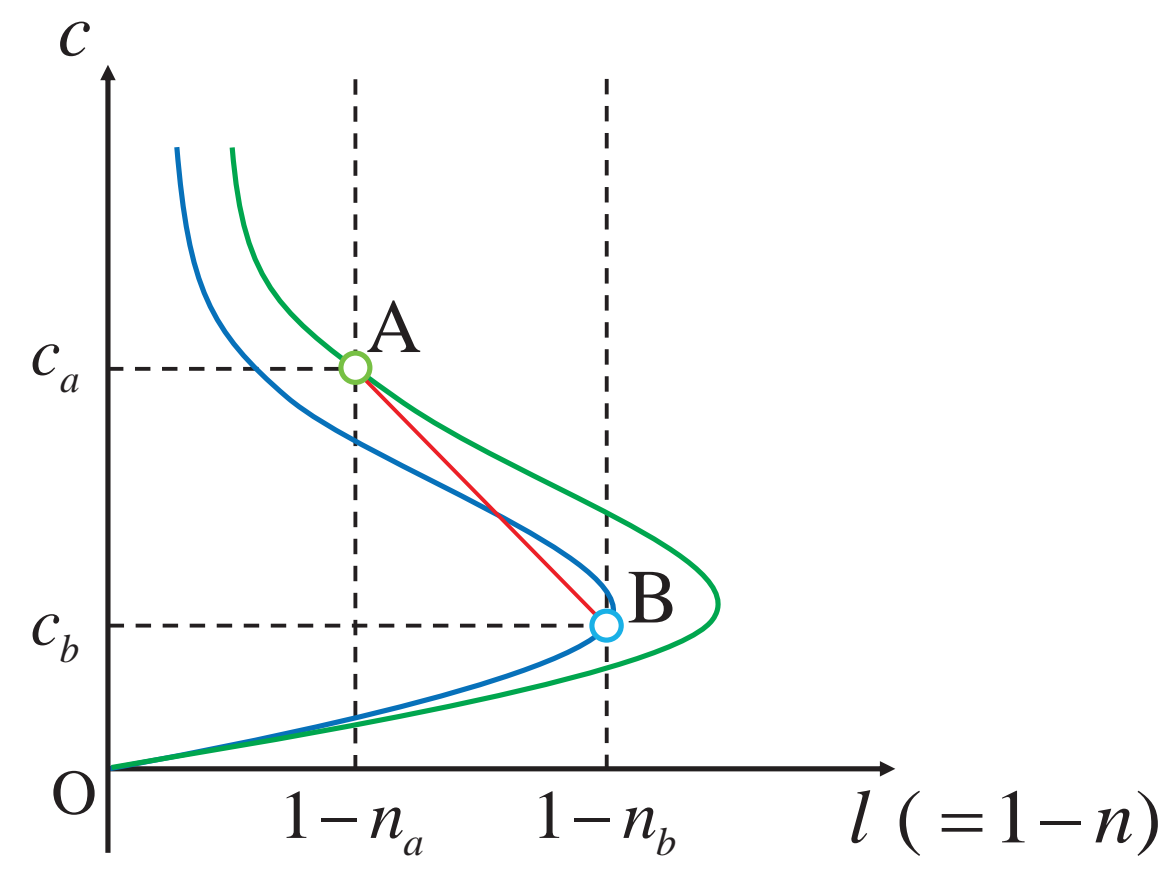

Figure 5: Income expansion paths with leisure being inferior

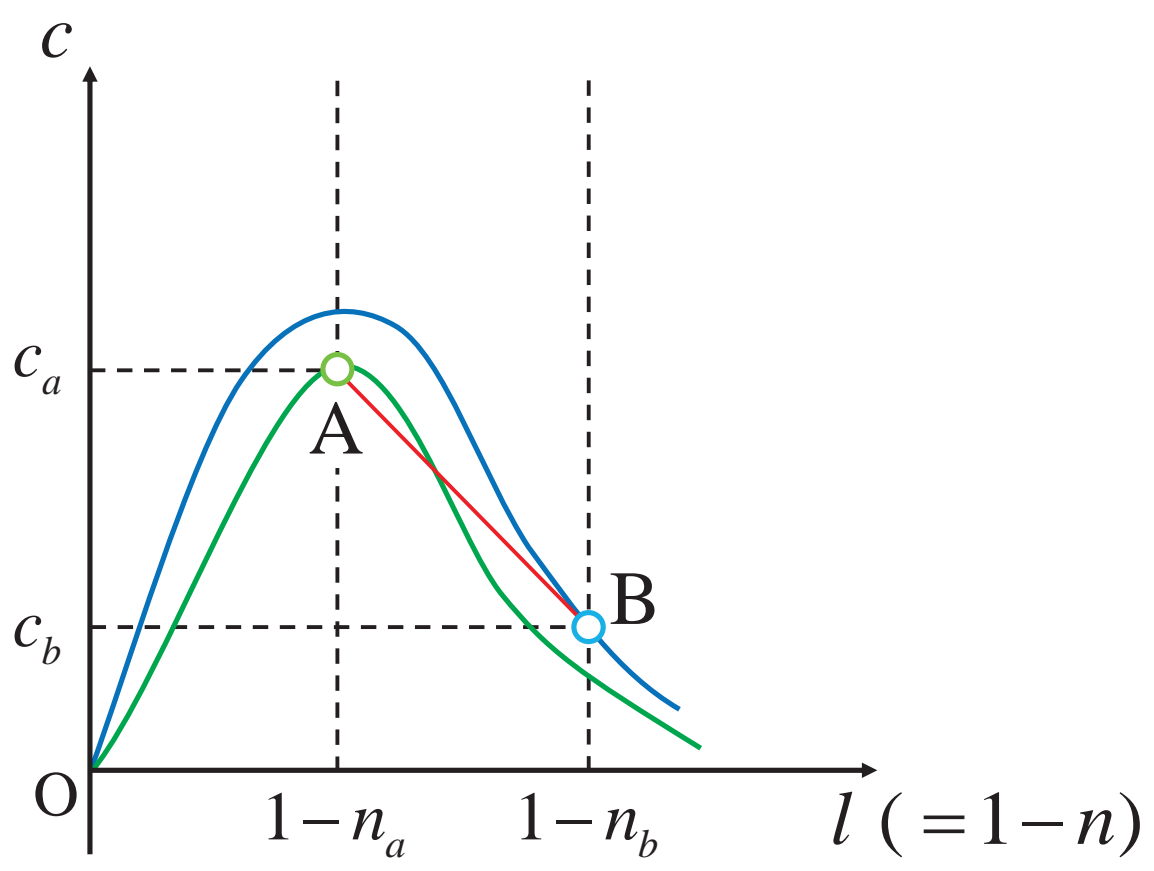

Figure 6: Income expansion paths with consumption being inferior 


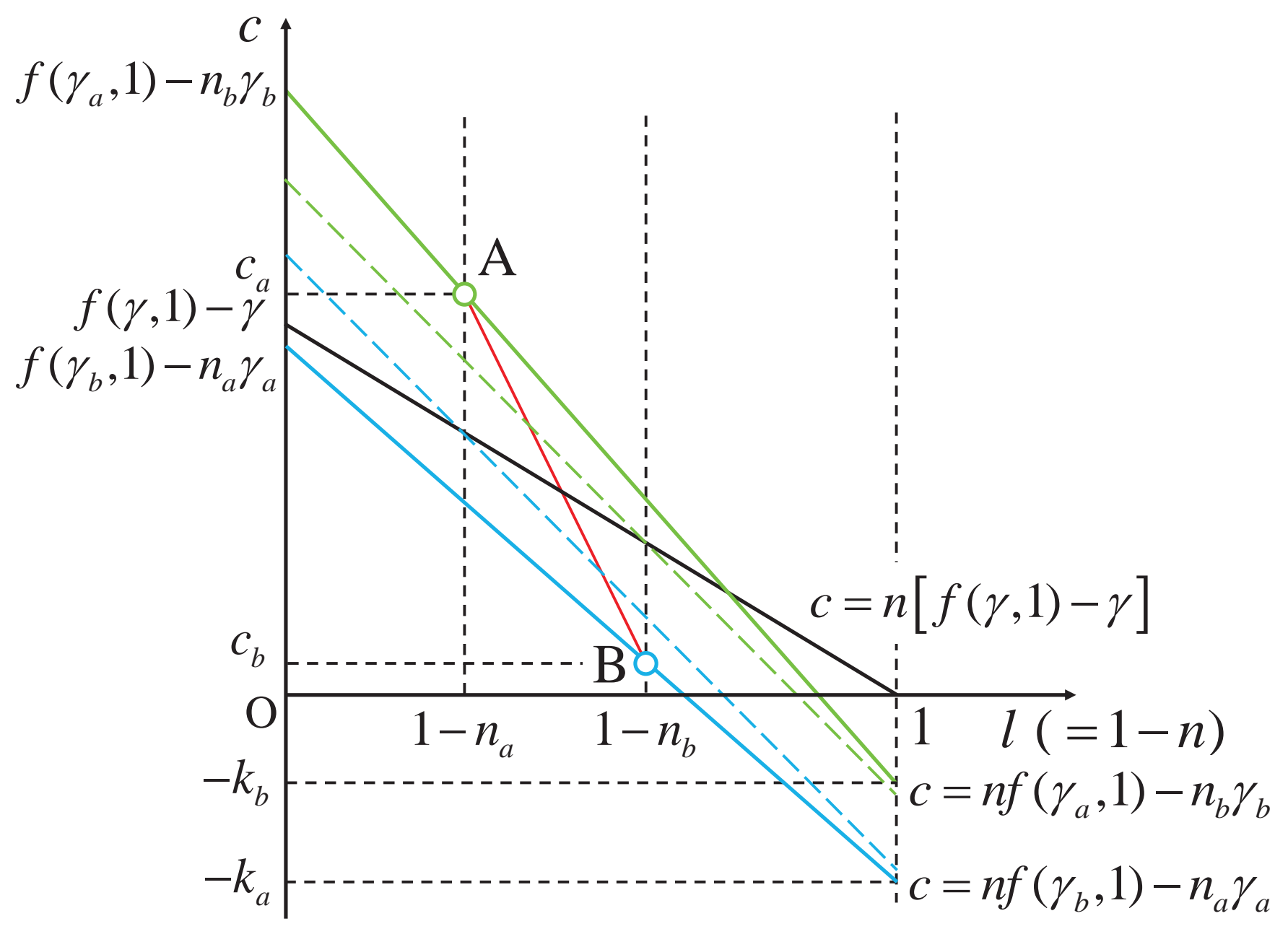

Figure 7: Pairs $\left(c_{a}, l_{a}\right)$ and $\left(c_{b}, l_{b}\right)$ with $\gamma_{a}>\gamma>\gamma_{b}$

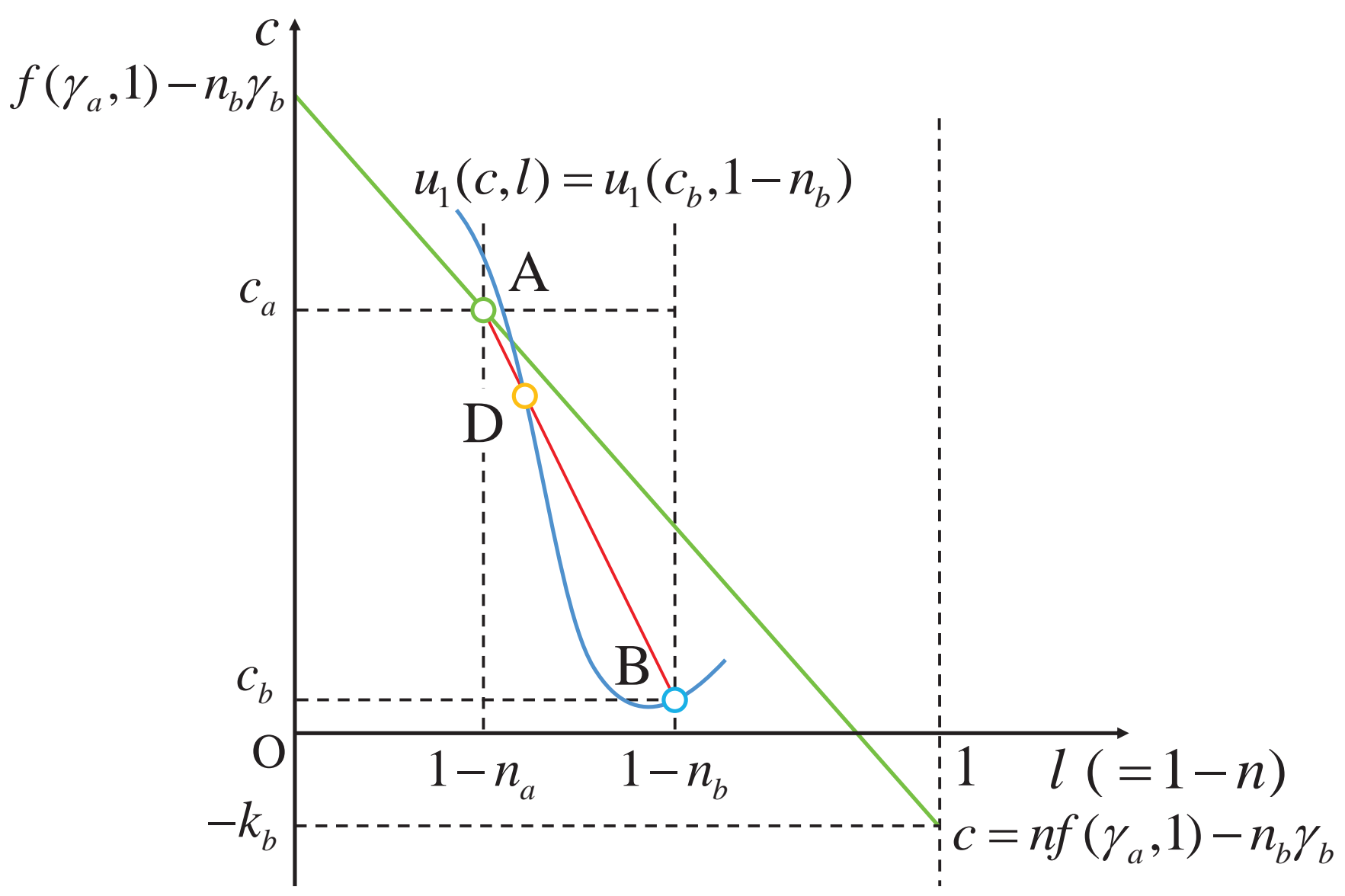

Figure 8: The locus of $u_{1}(c, l)=u_{1}\left(c_{b}, l_{b}\right)$ 